\title{
AN ESTIMATE OF THE RATE OF CONVERGENCE OF AN APPROXIMATING SCHEME APPLIED TO A STOCHASTIC DIFFERENTIAL EQUATION WITH AN ADDITIONAL PARAMETER
}

UDC 519.21

\author{
YU. S. MISHURA AND A. V. SHVA Ĭ
}

\begin{abstract}
We consider a stochastic differential equation with a diffusion coefficient involving an additional process viewed as a parameter. Given a rate of convergence of the Euler approximations for this parameter, we find the mean square rate of convergence of the Euler approximation scheme. An example is considered where the parameter is driven by a fractional Brownian motion.
\end{abstract}

\section{INTRODUCTION}

We consider functions $f(t, x):[0, T] \times \mathbb{R} \rightarrow \mathbb{R}$ and $g(t, v, x):[0, T] \times \mathbb{R} \times \mathbb{R} \rightarrow \mathbb{R}$ that are measurable with respect to all their arguments. We assume that the following linear growth condition holds for the functions $f$ and $g$ :

$$
|f(t, x)| \leq C(1+|x|), \quad|g(t, v, x)| \leq C(1+|x|) .
$$

We also assume that the Lipschitz conditions hold for $f$ and $g$ :

$$
\begin{gathered}
|f(t, x)-f(s, y)| \leq C(|t-s|+|x-y|), \\
|g(t, v, x)-g(s, w, y)| \leq C(|t-s|+|v-w|+|x-y|),
\end{gathered}
$$

where $C$ is a certain constant.

Consider a complete probability space $\left(\Omega, \mathcal{F}, \mathcal{F}_{t}, \mathrm{P}\right)$ with a filtration satisfying the standard conditions. Let $\left\{W_{t}, \mathcal{F}_{t}, t \in[0, T]\right\}$ be a standard Wiener process and let $\left\{\sigma_{t}, \mathcal{F}_{t}, t \in[0, T]\right\}$ be a stochastic process adapted to the filtration $\left\{\mathcal{F}_{t}, t \in[0, T]\right\}$.

We study the following stochastic differential equation:

$$
d X_{t}=f\left(t, X_{t}\right) d t+g\left(t, \sigma_{t}, X_{t}\right) d W_{t}, \quad X_{0}=x_{0} \in \mathbb{R}, t \in[0, T] .
$$

We treat $\sigma_{t}$ here as a parameter and say that (3) is an equation with an additional parameter. Assume that the parameter satisfies the Hölder condition with index $\alpha \in(0,1)$, that is,

$$
\left|\sigma_{t}-\sigma_{s}\right| \leq \widehat{C}_{1}(\omega)|t-s|^{\alpha},
$$

where $\widehat{C}_{1}(\omega)$ is a random variable that is finite with probability one.

2010 Mathematics Subject Classification. Primary 60H10.

Key words and phrases. Fractional Brownian motion, approximate solution of stochastic differential equations.

The first author is indebted to the European Commission for support in the framework of the "Marie Curie Actions" program, grant PIRSES-GA-2008-230804.

The second author, Alexander Shval̆, died tragically on October 10, 2010, after the original Ukrainian issue had already been published. 
Equation (3) is well studied for the case in which the function $g\left(t, \sigma_{t}, X_{t}\right)$ does not depend on $\sigma_{t}$. In particular, it has a unique solution that can be approximated in the space $L_{p}(\Omega \times[0, T])$ with as good accuracy as one wants. The rate of convergence of Euler approximations is $\delta^{1 / 2}$, where $\delta$ is the diameter of a partition (see, for example, [2] or (4)). More accurate approximation schemes are considered in [5].

On the other hand, quite a lot of literature is devoted to processes with switching. In our language, switching is an additional parameter. In particular, Markov and semiMarkov switchings are considered in the papers [6]- $[$.

In the framework of problems of financial mathematics, equation (3) can be viewed as an equation determining the price of a risky asset with a stochastic volatility $g\left(t, \sigma_{t}, X_{t}\right)$, where the additional parameter $\sigma_{t}$ models an extra randomness due to the action of exterior forces. In its turn, the parameter $\sigma_{t}$ can be modeled either by a diffusion process [10] or a fractional diffusion process. The latter is defined as a stochastic process with a fractional Brownian component and with Hurst index $H \in(1 / 2,1]$ corresponding to stochastic processes with long memory.

The paper is organized as follows. We prove in Section 2 that equation (3) possesses a unique solution. Euler approximations for equation (3) are constructed in Section 3. A theorem on the rate of convergence of Euler approximations is also proved in Section 3 (the rate of convergence turns out to be $\delta^{\min \{\alpha, \beta, 1 / 2\}}$, where $\delta$ is the diameter of the partition and where the index $\beta$ corresponds to the mean square rate of convergence for $\sigma_{t}$; see condition (91)). Section 4 contains an example of an equation with an additional parameter determined as a solution of a stochastic differential equation driven by a fractional Brownian motion with Hurst index $H \in(1 / 2,1)$. We show in this case that the rate of convergence is $\delta^{\min \{2 H-1,1 / 2\}}$.

\section{Existence AND Uniqueness of a SOlution of AN EQUATion WITH AN ADDITIONAL PARAMETER}

First we show that equation (3) with an additional parameter $\sigma_{t}$ possesses a unique solution in the space $L_{2}(\Omega \times[0, T])$.

Lemma 2.1. Let $X=\left\{X_{t}, t \geq 0\right\}$ be a nonanticipating stochastic process with respect to the filtration $\left\{\mathcal{F}_{t}, t \geq 0\right\}$. Moreover, we assume that $X \in L_{2}(\Omega \times[0, T])$.

Consider the operator

$$
(A X)_{t}=x_{0}+\int_{0}^{t} f\left(s, X_{s}\right) d s+\int_{0}^{t} g\left(s, \sigma_{s}, X_{s}\right) d W_{s},
$$

where $x_{0} \in \mathbb{R}, \sigma_{t}$ is an $\mathcal{F}_{t}$-adapted process satisfying condition (4), and where $f$ and $g$ are some functions such that conditions (1) and (2) hold.

Then $A$ is a contracting operator in the space $L_{2}^{k}(\Omega \times[0, T])$ equipped with the norm

$$
\|X\|_{k}^{2}=\int_{0}^{T} e^{-k t} \mathrm{E} X_{t}^{2} d t .
$$

Proof. Note that the integrals on the right-hand side of (15) are well defined. This follows, since $X$ is a nonanticipating process and in view of conditions (1) and (4). We need to prove that there are $0<\lambda<1$ and $k>0$ such that

$$
\|A(X-Y)\|_{k}^{2} \leq \lambda\|X-Y\|_{k}^{2}
$$

for all nonanticipating stochastic processes $X, Y \in L_{2}^{k}(\Omega \times[0, T])$. 
We rewrite the left-hand side of inequality (6) as follows:

$$
\begin{aligned}
&\|A(X-Y)\|_{k}^{2}=\int_{0}^{T} e^{-k t} \mathrm{E}\left(\int_{0}^{t}\left(f\left(s, X_{s}\right)-f\left(s, Y_{s}\right)\right) d s\right.\left.\quad+\int_{0}^{t}\left(g\left(t, \sigma_{s}, X_{s}\right)-g\left(t, \sigma_{s}, Y_{s}\right)\right) d W_{s}\right)^{2} d t \\
& \leq \int_{0}^{T} e^{-k t}\left(2 \mathrm{E}\left(\int_{0}^{t}\left(f\left(s, X_{s}\right)-f\left(s, Y_{s}\right)\right) d s\right)^{2}\right. \\
&\left.\quad+2 \int_{0}^{t} \mathrm{E}\left(g\left(t, \sigma_{s}, X_{s}\right)-g\left(t, \sigma_{s}, Y_{s}\right)\right)^{2} d s\right) d t \\
& \leq 2 T\left\|\int_{0}^{t}\left(f\left(s, X_{s}\right)-f\left(s, Y_{s}\right)\right) d s\right\|_{k}^{2} \\
&+2\left\|\int_{0}^{t}\left(g\left(t, \sigma_{s}, X_{s}\right)-g\left(t, \sigma_{s}, Y_{s}\right)\right) d W_{s}\right\|_{k}^{2} .
\end{aligned}
$$

Now we estimate each term separately. In doing so, we use the inequality

$$
\mathrm{E}\left(\int_{0}^{T}\left|X_{s}\right| d s\right)^{2} \leq T \int_{0}^{T} \mathrm{E}\left|X_{s}\right|^{2} d s
$$

together with property (2) for the functions $f(t, x)$ and $g(t, v, x)$ :

$$
\begin{aligned}
\left\|\int_{0}^{t}\left(f\left(s, X_{s}\right)-f\left(s, Y_{s}\right)\right) d s\right\|_{k}^{2} \leq & \left\|\int_{0}^{t} C\left(\left|X_{s}-Y_{s}\right|\right) d s\right\|_{k}^{2} \\
\leq & \int_{0}^{T} C^{2} e^{-k t} \mathrm{E}\left(\int_{0}^{t}\left|X_{s}-Y_{s}\right| d s\right)^{2} d t \\
\leq & T C^{2} \int_{0}^{T} e^{-k t} \int_{0}^{t} \mathrm{E}\left|X_{s}-Y_{s}\right|^{2} d s d t \\
& +T C^{2} \int_{0}^{T} \mathrm{E}\left|X_{s}-Y_{s}\right|^{2} \int_{s}^{T} e^{-k t} d t d s \\
\leq & T C^{2} / k \cdot\left\|X_{s}-Y_{s}\right\|_{k}^{2} .
\end{aligned}
$$

The bounds for $g(t, v, x)$ can be derived similarly. Combining both results we conclude that

$$
\begin{gathered}
\left\|\int\left(f\left(s, X_{s}\right)-f\left(s, Y_{s}\right)\right) d s\right\|_{k}^{2} \leq C_{k} \cdot\|X-Y\|_{k}^{2}, \\
\left\|\int\left(g\left(s, v_{s}, X_{s}\right)-g\left(s, v_{s}, Y_{s}\right)\right) d W_{s}\right\|_{k}^{2} \leq C_{k} \cdot\|X-Y\|_{k}^{2},
\end{gathered}
$$

where $C_{k}=C / k \rightarrow 0$ as $k \rightarrow \infty$.

Relations (77) yield $\|(A(X-Y))\|_{k}^{2} \leq 2 C_{k} \cdot\|X-Y\|_{k}^{2}$. Since one can always pick $k$ in such a way that $C_{k}<1 / 2$, inequality (6) is proved.

Remark 2.1. Lemma 2.1 means that there exists a space $L_{2}^{k}(\Omega \times[0, T])$ where equation (3) possesses a unique solution. The norm $\|\cdot\|_{k}$ is equivalent to the standard norm in $L_{2}(\Omega \times[0, T])$, whence we conclude that the equation possesses a unique solution in the space $L_{2}(\Omega \times[0, T])$ equipped with the standard norm. It is worth mentioning that the 
only properties used in the proof above are the continuity of trajectories of the process $\sigma_{t}$ and that $\sigma_{t}$ is adapted with the main filtration needed for the stochastic integral to be well defined.

Corollary 2.1. The above theorem holds if $X_{0}$ is an $\mathcal{F}_{0}$-measurable random variable. Moreover, if additionally $\mathrm{E}\left|X_{0}\right|^{m}<\infty$ for some $m>0$, then $\mathrm{E}\left|X_{t}\right|^{m}<\infty$ for all $t \in[0, T]$.

We will use the latter result in what follows.

\section{Rate of CONVERGEnCE of An Approximate Solution of a STOCHAStic DIFFERENTIAL EQUATION WITH AN ADDITIONAL PARAMETER}

We apply the Euler method to get an approximate solution of equation (3). According to the method, we introduce a uniform partition $0=\tau_{0}<\tau_{1}<\cdots<\tau_{n}=T$ of the interval $[0, T]$. The diameter of the partition is denoted by $\delta=T / n, \tau_{k}=k \delta$.

Note that the assumption that the partition is uniform makes our reasoning easier; however, the result holds in the general case.

We define the process $X_{t}^{\delta}$ at the points of the partition as follows:

$$
X_{\tau_{n+1}}^{\delta}=X_{\tau_{n}}^{\delta}+f\left(\tau_{n}, X_{\tau_{n}}^{\delta}\right)\left(\tau_{n+1}-\tau_{n}\right)+g\left(\tau_{n}, \sigma_{\tau_{n}}^{\delta}, X_{\tau_{n}}^{\delta}\right)\left(W_{\tau_{n+1}}-W_{\tau_{n}}\right),
$$

where $X_{\tau_{0}}^{\delta}=X_{0}^{\delta}$ and where $\sigma_{t}^{\delta}$ is an approximation of the process $\sigma_{t}$ defined on the same partition.

Let the rate of convergence of $\sigma_{t}^{\delta}$ to $\sigma_{t}$ be such that

$$
\mathrm{E}\left|\sigma_{t}-\sigma_{t}^{\delta}\right|^{2} \leq C \delta^{2 \beta} \quad \text { for some } \beta>0 .
$$

Put $t_{u}=\max \left\{\tau_{n}: \tau_{n}<u\right\}$.

According to the method, the continuous iteration of the Euler approximation of the process $X_{u}$ is given by

$$
X_{u}^{\delta}=X_{t_{u}}^{\delta}+f\left(t_{u}, X_{t_{u}}^{\delta}\right) \cdot\left(u-t_{u}\right)+g\left(t_{u}, \sigma_{t_{u}}^{\delta}, X_{t_{u}}^{\delta}\right)\left(W_{u}-W_{t_{u}}\right)
$$

or, using the stochastic integral,

$$
X_{u}^{\delta}=X_{0}^{\delta}+\int_{0}^{u} f\left(t_{s}, X_{t_{s}}^{\delta}\right) d s+\int_{0}^{u} g\left(t_{s}, \sigma_{t_{s}}^{\delta}, X_{t_{s}}^{\delta}\right) d W_{s} .
$$

Lemma 3.1. Let the coefficients of equation (3) satisfy conditions (1) and (2). Assume that $\mathrm{E}\left|X_{0}^{\delta}\right|^{m}<\infty$ for some $m>0$. Then $\mathrm{E}\left|X_{u}^{\delta}\right|^{m}<\infty$ for all $u \in[0, T]$.

Proof. First we prove the lemma for the points of the partition. We use the method of mathematical induction. Indeed, $\mathrm{E}\left|X_{0}^{\delta}\right|^{m}<\infty$ by the assumptions of the lemma. Let $\mathrm{E}\left|X_{\tau_{n}}^{\delta}\right|^{m}<\infty$. Then definition (8) implies that

$$
\begin{aligned}
\mathrm{E}\left|X_{\tau_{n+1}}^{\delta}\right|^{m} & =\mathrm{E}\left|X_{\tau_{n}}^{\delta}+f\left(\tau_{n}, X_{\tau_{n}}^{\delta}\right) \delta+g\left(\tau_{n}, \sigma_{\tau_{n}}^{\delta}, X_{\tau_{n}}^{\delta}\right)\left(W_{\tau_{n+1}}-W_{\tau_{n}}\right)\right|^{m} \\
& \leq 3^{m} \mathrm{E}\left|X_{\tau_{n}}^{\delta}\right|^{2}+3^{m} C^{m} \delta^{m} \mathrm{E}\left(1+\left|X_{\tau_{n}}^{\delta}\right|\right)^{m}+3^{m} C^{m} \delta^{m / 2} \mathrm{E}\left(1+\left|X_{\tau_{n}}^{\delta}\right|\right)^{m} \\
& <\infty .
\end{aligned}
$$

Using definition (10), we check that the same bound holds for all other points of the interval $[0, T]$.

Theorem 3.1. Assume that conditions (1), (2), (4), and (9) hold. Let $X_{0}^{\delta}=X_{0}$ for all $\delta>0$ and let $\mathrm{E}\left|X_{0}\right|^{2}<\infty$. Further we assume that there exists a constant $C>0$ such that $\mathrm{E} \widehat{C}_{1}(\omega)^{2} \leq C$, where $\widehat{C}_{1}(\omega)$ is the random variable defined in condition (4). 
Then the approximation of the process (11) converges to the process (3) in the mean square sense. Moreover,

$$
\mathrm{E}\left|X_{u}-X_{u}^{\delta}\right|^{2} \leq C\left(\delta^{2 \alpha}+\delta^{2 \beta}+\delta^{2}+\delta\right) \exp (C T)
$$

where $C$ is some constant and where $\alpha$ and $\beta$ are Hölder exponents involved in conditions (4) and (9), respectively.

Proof. We deduce from equalities (3) and (11) that

$$
\begin{aligned}
& \left|X_{u}-X_{u}^{\delta}\right| \\
& \quad \leq \int_{0}^{u}\left|f\left(s, X_{s}\right)-f\left(t_{s}, X_{t_{s}}^{\delta}\right)\right| d s+\left|\int_{0}^{u} g\left(s, \sigma_{s}, X_{s}\right)-g\left(t_{s}, \sigma_{t_{s}}^{\delta}, X_{t_{s}}^{\delta}\right) d W_{s}\right| .
\end{aligned}
$$

Then we estimate the second moment of the first integral on the right-hand side of the latter relation:

$$
\begin{aligned}
& \mathrm{E}\left(\int_{0}^{u}\left|f\left(s, X_{s}\right)-f\left(t_{s}, X_{t_{s}}^{\delta}\right)\right| d s\right)^{2} \\
& \quad \leq T \int_{0}^{u} \mathrm{E}\left(C\left|s-t_{s}\right|+C\left|X_{s}-X_{t_{s}}^{\delta}\right|\right)^{2} d s \\
& \quad \leq 2 C \cdot T \delta^{2}+2 C T \cdot \int_{0}^{u} \mathrm{E}\left(\left|X_{s}-X_{s}^{\delta}\right|+\left|X_{s}^{\delta}-X_{t_{s}}^{\delta}\right|\right)^{2} d s .
\end{aligned}
$$

The second moment of the second integral is estimated similarly:

$$
\begin{aligned}
& \mathrm{E}\left(\int_{0}^{u}\left(g\left(s, \sigma_{s}, X_{s}\right)-g\left(t_{s}, \sigma_{t_{s}}^{\delta}, X_{t_{s}}^{\delta}\right)\right) d W_{s}\right)^{2} \\
& \leq \int_{0}^{u}\left(3 C^{2} \cdot\left|s-t_{s}\right|^{2}+3 C^{2} \cdot \mathrm{E}\left|\sigma_{s}-\sigma_{t_{s}}^{\delta}\right|^{2}+3 C^{2} \cdot \mathrm{E}\left|X_{s}-X_{t_{s}}^{\delta}\right|^{2}\right) d s \\
& \leq \int_{0}^{u}\left(3 C^{2} \cdot \delta^{2}+6 C^{2} \cdot \mathrm{E}\left|\sigma_{s}-\sigma_{t_{s}}\right|^{2}+6 C^{2} \cdot \mathrm{E}\left|\sigma_{t_{s}}-\sigma_{t_{s}}^{\delta}\right|^{2}\right. \\
& \left.+6 C^{2} \cdot \mathrm{E}\left|X_{s}-X_{s}^{\delta}\right|^{2}+6 C^{2} \cdot \mathrm{E}\left|X_{s}^{\delta}-X_{t_{s}}^{\delta}\right|^{2}\right) d s .
\end{aligned}
$$

This bound together with (4) and (9) implies that

$$
\begin{aligned}
\mathrm{E}\left(\int_{0}^{u}\right. & \left.\left(g\left(s, \sigma_{s}, X_{s}\right)-g\left(t_{s}, \sigma_{t_{s}}^{\delta}, X_{t_{s}}^{\delta}\right)\right) d W_{s}\right)^{2} \\
\leq & 3 C^{2} T \cdot \delta^{2}+6 C^{2} \mathrm{E} C_{1}(\omega)^{2} T \cdot \delta^{2 \alpha}+6 C^{2} T \cdot \delta^{2 \beta} \\
\quad & +6 C^{2} \int_{0}^{u}\left(\mathrm{E}\left|X_{s}-X_{s}^{\delta}\right|^{2}+\mathrm{E}\left|X_{s}^{\delta}-X_{t_{s}}^{\delta}\right|^{2}\right) d s .
\end{aligned}
$$

Here and in what follows the same symbol $C$ denotes different constants whose exact values do not matter for the reasoning in different places; moreover, the values of the constant may change from place to place. Combining bounds (13)-(15) we obtain

$$
\mathrm{E}\left|X_{u}-X_{u}^{\delta}\right|^{2} \leq C \delta^{2}+C \delta^{2 \alpha}+C \delta^{2 \beta}+C \int_{0}^{u}\left(\mathrm{E}\left|X_{s}-X_{s}^{\delta}\right|^{2}+\mathrm{E}\left|X_{s}^{\delta}-X_{t_{s}}^{\delta}\right|^{2}\right) d s
$$

Next we estimate $\mathrm{E}\left|X_{s}^{\delta}-X_{t_{s}}^{\delta}\right|^{2}$. Using equation (10) we deduce that

$$
\begin{aligned}
\mathrm{E}\left|X_{s}^{\delta}-X_{t_{s}}^{\delta}\right|^{2} & \leq \mathrm{E}\left(f\left(t_{s}, X_{t_{s}}^{\delta}\right) \cdot\left(s-t_{s}\right)+g\left(t_{s}, \sigma_{t_{s}}^{\delta}, X_{t_{s}}^{\delta}\right)\left(W_{s}-W_{t_{s}}\right)\right)^{2} \\
& \leq C \delta^{2} \mathrm{E}\left(1+\left|X_{t_{s}}^{\delta}\right|\right)^{2}+C \delta \mathrm{E}\left(1+\left|X_{t_{s}}^{\delta}\right|\right)^{2} \\
& \leq C\left(\delta+\delta^{2}\right) \mathrm{E}\left(1+\left|X_{t_{s}}^{\delta}\right|\right)^{2}
\end{aligned}
$$


whence, by representation (11),

$$
\begin{aligned}
\mathrm{E}\left(1+\left|X_{t_{u}}^{\delta}\right|\right)^{2} & \leq 2+2 \mathrm{E}\left(X_{0}+\int_{0}^{t_{u}} f\left(t_{s}, X_{t_{s}}^{\delta}\right) d s+\int_{0}^{t_{u}} g\left(t_{s}, \sigma_{t_{s}}^{\delta}, X_{t_{s}}^{\delta}\right) d W_{s}\right)^{2} \\
& <2+6 \mathrm{E} X_{0}^{2}+6(T+1) \int_{0}^{t_{u}} \mathrm{E}\left(1+\left|X_{t_{s}}^{\delta}\right|\right)^{2} d s .
\end{aligned}
$$

Now Lemma 3.1 implies that the right-hand side of (18) is finite. This allows one to apply the Gronwall inequality and this yields

$$
\mathrm{E}\left(1+\left|X_{t_{u}}^{\delta}\right|\right)^{2}<\left(2+6 X_{0}^{2}\right) \exp (6 T(T+1))
$$

Then (17) and (19) imply that

$$
\mathrm{E}\left|X_{s}^{\delta}-X_{t_{s}}^{\delta}\right|^{2} \leq C\left(\delta+\delta^{2}\right) \exp (C T) .
$$

Finally, (16), (20), and the Gronwall inequality prove that

$$
\mathrm{E}\left|X_{u}-X_{u}^{\delta}\right|^{2} \leq\left(C \delta^{2}+6 C^{2} \mathrm{E} C_{1}(\omega)^{2} T \delta^{2 \alpha}+6 C^{2} T \delta^{2 \beta}+C \cdot \delta\right) \exp (C T)
$$

The latter inequality means that the Euler approximations converge in the mean square sense to the solutions of equation (3). Passing to the square roots of both sides of (21), we find that the rate of convergence is $O\left(\delta^{\min \{\alpha, \beta, 1 / 2\}}\right)$.

\section{The CASE Where the ADditional PARAMETER IS DRIVEN BY A FRACTIONAL BROWNIAN MOTION}

We studied the approximation scheme for the general case in the preceding sections. Now we consider a particular case of an additional parameter. We check that conditions (44) and (9) hold in this particular case and then we obtain the rate of convergence in the general scheme.

Definition 4.1. A Gaussian process $B_{t}^{H}$ such that $B_{0}^{H}=0$, E $B_{t}^{H}=0$, and

$$
\mathrm{E} B_{t}^{H} B_{s}^{H}=\frac{1}{2}\left(|s|^{2 H}+|t|^{2 H}-|t-s|^{2 H}\right)
$$

is called a fractional Brownian motion $B_{t}^{H}=\left\{B_{t}^{H}, \mathcal{F}_{t}, t \geq 0\right\}$ with the Hurst index $H \in(0,1)$.

In what follows we consider a continuous version of this process that exists by the Kolmogorov theorem.

If $H=1 / 2$, then the fractional Brownian motion coincides with a standard Wiener process. If $H \neq 1 / 2$, then the fractional Brownian motion is a process with a "memory". If $H \in(1 / 2,1)$, then we deal with the phenomenon of the so-called "long memory". Long memory processes are widely used in mathematical modeling and in finance mathematics. A model with a fractional Brownian motion is believed to adequately describe the evolution of prices of a risky asset with random volatility. It is the volatility that possesses the property of long memory.

Consider a measurable function $a(t, x):[0, T] \times \mathbb{R} \rightarrow \mathbb{R}$ satisfying the following conditions:

1) local Hölder property with respect to spatial arguments: there exists a constant $C_{R}>0$ such that

$$
|a(t, x)-a(t, y)| \leq C_{R}|x-y|
$$

for all $t \geq 0$ and $|x|,|y| \leq R$; 
2) the Hölder property with respect to time: there are constants $C>0$ and $\theta$ such that $2 H-1 \leq \theta \leq 1$ and

$$
|a(t, x)-a(s, x)| \leq C|s-t|^{\theta}
$$

for all $t, s \in[0, T]$

3) boundedness: $|a(t, x)| \leq A$ for some constant $A$.

Consider another measurable function $b(t, x):[0, T] \times \mathbb{R} \rightarrow \mathbb{R}$ satisfying the following conditions:

4) $b(t, x)$ is differentiable with respect to $x$, and the Lipschitz condition holds for the spatial argument

$$
\exists C>0: \quad|b(t, x)-b(t, y)| \leq C|x-y|
$$

5) the partial derivative of $b$ with respect to $x$ is locally Hölderian; that is, there exists a constant $C_{R}>0$ such that

$$
\left|b_{x}(t, x)-b_{x}(t, y)\right| \leq C_{R}|x-y|
$$

for all $t \geq 0$ and $|x|,|y| \leq R$;

6) the functions $b$ and $b_{x}$ satisfy the Hölder condition with respect to time; that is, there exists $\gamma$ such that $1-H<\gamma \leq 1$ and

$$
|b(t, x)-b(s, x)|+\left|b_{x}(t, x)-b_{x}(s, x)\right| \leq C|t-s|^{\gamma}
$$

for all $t$ and $x, y$;

7) the function $b(t, x)$ is bounded: $|b(t, x)| \leq B$ for some constant $B$.

Now the process $\sigma_{t}$ is defined as a solution of the following stochastic differential equation:

$$
d \sigma_{t}=a\left(t, \sigma_{t}\right) d t+b\left(t, \sigma_{t}\right) d B_{t}^{H}
$$

with the initial condition $\sigma_{0} \in \mathbb{R}$, where $B_{t}^{H}$ is the fractional Brownian motion with the Hurst index $H \in(1 / 2 ; 1)$. We rewrite equation (22) in the integral form:

$$
\sigma_{t}=\sigma_{0}+\int_{0}^{t} a\left(s, \sigma_{s}\right) d s+\int_{0}^{t} b\left(s, \sigma_{s}\right) d B_{s}^{H},
$$

where the integral $\int_{0}^{t} b\left(s, \sigma_{s}\right) d B_{s}^{H}$ is understood pathwise (see [3]).

It is proved in [3] that equation (23) has a unique continuous solution if conditions 1) -7 ) hold. Thus $\sigma_{t}$ is well defined.

Consider a partition $0=\tau_{0}<\tau_{1}<\cdots<\tau_{n}=T$ described in the latter section. Using recurrence we define the values of the Euler approximation of a solution of equation (23) at the nodes of the partition:

$$
\sigma_{\tau_{k+1}}^{\delta}=\sigma_{\tau_{k}}^{\delta}+a\left(\tau_{k}, \sigma_{\tau_{k}}^{\delta}\right) \delta+b\left(\tau_{k}, \sigma_{\tau_{k}}^{\delta}\right)\left(B_{\tau_{k+1}}^{H}-B_{\tau_{k}}^{H}\right) .
$$

The continuous approximation is given by

$$
\sigma_{t}^{\delta}=\sigma_{0}^{\delta}+\int_{0}^{t} a\left(\theta_{u}, \sigma_{\theta_{u}}^{\delta}\right) d u+\int_{0}^{t} b\left(\theta_{u}, \sigma_{\theta_{u}}^{\delta}\right) d B_{u}^{H},
$$

where $\theta_{u}=\max \left\{\tau_{n}: \tau_{n}<u\right\}$.

Theorem 4.1. Assume that conditions 1)-7) hold. Then

$$
\left|\sigma_{t}^{\delta}\right| \leq C(\omega) ; \quad\left|\sigma_{s}^{\delta}-\sigma_{r}^{\delta}\right| \leq C(\omega)|s-r|^{H-\varepsilon}
$$

for all $0<\varepsilon<H$, where $C(\omega)$ is a square integrable random variable, that is,

$$
\mathrm{E} C(\omega)^{2}<\infty .
$$


YU. S. MISHURA AND A. V. SHVA I

Proof. Inequalities (26) are proved in [1]. It remains to estimate the second moment $\mathrm{E} C(\omega)^{2}$.

Without loss of generality we assume that $\delta<1$. Then, for $0<\varepsilon<H$ and all $\beta \in(1-H, \gamma \wedge 1 / 2)$,

$$
\left|\int_{0}^{t} f(x) d B_{x}^{H}\right| \leq C_{1}(\omega)\left(\int_{0}^{t}|f(x)| x^{-\beta} d x+\int_{0}^{t} \int_{0}^{r} \frac{|f(r)-f(x)|}{(r-x)^{\beta+1}} d x d r\right)
$$

(see [3]), where

$$
C_{1}(\omega)=\frac{1}{\Gamma(\beta)} \sup _{0 \leq s<z \leq T}\left|D_{z-}^{1-\beta} B_{z-}^{H}(s)\right|
$$

and where $D_{z-}^{1-\beta}$ is the left fractional derivative of order $1-\beta$ of the fractional Brownian motion $B^{H}$. It is shown in [3] that

$$
C_{1}(\omega):=C_{\alpha, H, \varepsilon}\left(\int_{0}^{T} \int_{0}^{T} \frac{\left|B_{x}^{H}-B_{y}^{H}\right|^{2 / \varepsilon}}{|x-y|^{2 H / \varepsilon}} d x d y\right)^{\varepsilon / 2} \leq C_{\alpha, H, \varepsilon} \xi^{\varepsilon / 2} .
$$

Here $C_{\alpha, H, \varepsilon}$ denotes a certain constant and the random variable $\xi$ on the right-hand side of (28) possesses all the moments. Now equality (25) and bound (27) imply that

$$
\begin{aligned}
\left|\sigma_{t}^{\delta}\right| \leq & \left|\sigma_{0}^{\delta}\right|+\int_{0}^{t}\left|a\left(\theta_{u}, \sigma_{\theta_{u}}^{\delta}\right)\right| d u+\left|\int_{0}^{t} b\left(\theta_{u}, \sigma_{\theta_{u}}^{\delta}\right) d B_{u}^{H}\right| \\
\leq & \left|\sigma_{0}^{\delta}\right|+A t+C_{1}(\omega) \int_{0}^{t} B u^{-\beta} d u+C_{1}(\omega) \int_{0}^{t} \int_{0}^{r} \frac{\left|b\left(\theta_{r}, \sigma_{\theta_{r}}^{\delta}\right)-b\left(\theta_{u}, \sigma_{\theta_{u}}^{\delta}\right)\right|}{(r-u)^{\beta+1}} d u d r \\
\leq & \left|\sigma_{0}^{\delta}\right|+B C_{1}(\omega) T^{1-\beta} /(1-\beta)+A T \\
& +C C_{1}(\omega) \int_{0}^{t} \int_{0}^{\theta_{r}} \frac{\left(\left|\theta_{r}-\theta_{u}\right|^{\gamma}+\left|\sigma_{\theta_{r}}^{\delta}-\sigma_{u}^{\delta}\right|+\left|\sigma_{\theta_{u}}^{\delta}-\sigma_{u}^{\delta}\right|\right)}{(r-u)^{\beta+1}} d u d r .
\end{aligned}
$$

It is known that

$$
\left|B_{t}^{H}-B_{s}^{H}\right| \leq C_{H, \varepsilon}|s-t|^{H-\varepsilon} \xi^{\varepsilon / 2}=C_{1}(\omega)|s-t|^{H-\varepsilon}
$$

(see [3]), where $C_{H, \varepsilon}$ is a certain positive constant and the random variable $\xi$ is defined in equality (28).

Using the preceding bounds we derive from equality (25) that

$$
\begin{aligned}
\left|\sigma_{u}^{\delta}-\sigma_{\theta_{u}}^{\delta}\right| & \leq \int_{\theta_{u}}^{u}\left|a\left(\theta_{u}, \sigma_{\theta_{u}}^{\delta}\right)\right| d u+\left|\int_{\theta_{u}}^{u} b\left(\theta_{u}, \sigma_{\theta_{u}}^{\delta}\right) d B_{u}^{H}\right| \\
& \leq A\left(u-\theta_{u}\right)+\left|b\left(\theta_{u}, \sigma_{\theta_{u}}^{\delta}\right)\right|\left|B_{u}^{H}-B_{\theta_{u}}^{H}\right| \\
& \leq A\left(u-\theta_{u}\right)+C_{1}(\omega) B\left(u-\theta_{u}\right)^{H-\varepsilon} \leq C_{2}(\omega)\left(u-\theta_{u}\right)^{H-\varepsilon}
\end{aligned}
$$

for all $\varepsilon \in(0, H)$, where $C_{2}(\omega)=B C_{1}(\omega)+A \delta^{1-H+\varepsilon}$. Now we estimate from above the right-hand side of (29). According to [1, (3.4.5)],

$$
P_{t}:=\int_{0}^{t} \int_{0}^{\theta_{r}}\left(\theta_{r}-\theta_{u}\right)^{\gamma}(r-u)^{-\beta-1} d u d r \leq C .
$$

Consider

$$
Q_{t}:=\int_{0}^{t} \int_{0}^{\theta_{r}}\left|\sigma_{\theta_{u}}^{\delta}-\sigma_{u}^{\delta}\right|(r-u)^{-\beta-1} d u d r .
$$

Using bounds (30) we obtain

$$
Q_{t} \leq C_{1}(\omega) \int_{0}^{t} \int_{0}^{\theta_{r}}\left(u-\theta_{u}\right)^{H-\varepsilon}(r-u)^{-\beta-1} d u d r \leq C_{1}(\omega) \delta^{H-\beta-\varepsilon} .
$$


Substituting the above estimates into (29), we get

$$
\begin{aligned}
\left|\sigma_{t}^{\delta}\right| \leq & \left|\sigma_{0}^{\delta}\right|+A T+B C_{1}(\omega) T^{1-\beta} /(1-\beta)+C C_{1}(\omega) Q_{t} \\
& +C Q_{T} P_{t}+C C_{1}(\omega) \int_{0}^{t} \int_{0}^{\theta_{r}} \varphi_{r, u} d u d r \\
\leq & C_{3}(\omega)+C C_{1}(\omega) \delta^{H-\beta-\varepsilon}+C C_{1}(\omega) \int_{0}^{t} \int_{0}^{\theta_{r}} \varphi_{r, u} d u d r
\end{aligned}
$$

where $\varphi_{r, u}:=\left|\sigma_{\theta_{r}}^{\delta}-\sigma_{u}^{\delta}\right|(r-u)^{-\beta-1}$ and

$$
C_{3}(\omega):=\left|\sigma_{0}^{\delta}\right|+A T+\frac{B C_{1}(\omega) T^{1-\beta}}{(1-\beta)}+C C_{1}(\omega)^{2} \delta^{H-\beta-\varepsilon} .
$$

Therefore

$$
\left|\sigma_{t}^{\delta}\right| \leq C_{4}(\omega)\left(1+\delta^{H-\beta-\varepsilon}+\int_{0}^{t} \int_{0}^{\theta_{r}} \varphi_{r, u} d u d r\right)
$$

where $C_{4}(\omega):=\max \left\{C_{3}(\omega), C C_{1}(\omega)\right\}$.

Reasoning similarly and applying (25) and (27) we estimate (36)

$$
\begin{aligned}
&\left|\sigma_{\theta_{s}}^{\delta}-\sigma_{u}^{\delta}\right| \leq C_{1}(\omega)\left(\int_{u}^{\theta_{s}} A d v+\int_{u}^{\theta_{s}} B(v-u)^{-\beta} d v\right. \\
&\left.\quad+\int_{u}^{\theta_{s}} \int_{u}^{\theta_{v}}\left|b\left(\theta_{v}, \sigma_{\theta_{v}}^{\delta}\right)-b\left(\theta_{z}, \sigma_{\theta_{z}}^{\delta}\right)\right|(v-z)^{-\beta-1} d z d v\right) \\
& \leq C_{2}(\omega)\left(\left(\theta_{s}-u\right)^{1-\beta}+\delta^{\gamma} \int_{u}^{\theta_{s}}\left(v-\theta_{v}\right)^{-\beta} d v+\int_{u}^{\theta_{s}} \int_{u}^{\theta_{v}} \varphi_{v, z} d z d v\right. \\
&\left.\quad+\int_{u}^{\theta_{s}} \int_{u}^{\theta_{v}}\left|\sigma_{z}^{\delta}-\sigma_{\theta_{z}}^{\delta}\right|(v-z)^{-\beta-1} d z d v\right) .
\end{aligned}
$$

Multiplying both sides of the latter inequality by $(s-u)^{-\beta-1}$ and then integrating over the interval $\left[0, \theta_{s}\right]$ we prove that

$$
\int_{0}^{\theta_{s}} \varphi_{s, u} d u \leq C_{2}(\omega) \sum_{i=1}^{4} Q_{s}^{i}
$$

where the $Q_{s}^{i}$ correspond to the terms on the right-hand side of (37). Every one of these terms is estimated separately. First,

$$
\begin{gathered}
Q_{s}^{1}:=\int_{0}^{\theta_{s}}\left(\theta_{s}-u\right)^{1-\beta}(s-u)^{-\beta-1} d u \leq C, \\
Q_{s}^{2}:=\delta^{\gamma} \int_{0}^{\theta_{s}} \int_{u}^{\theta_{s}}\left(v-\theta_{v}\right)^{-\beta} d v(s-u)^{-\beta-1} d u \\
\leq \beta^{-1} \delta^{\gamma} \int_{0}^{\theta_{s}}(s-v)^{-\beta}\left(v-\theta_{v}\right)^{-\beta} d v \leq C \delta^{\gamma-\beta} .
\end{gathered}
$$


Second,

$$
\begin{aligned}
Q_{s}^{3}:=\int_{0}^{\theta_{s}} \int_{u}^{\theta_{s}} \int_{u}^{\theta_{v}} \varphi_{v, z} d z d v(s-u)^{-\beta-1} d u \leq C \int_{0}^{\theta_{s}}(s-v)^{-\beta} \int_{0}^{\theta_{v}} \varphi_{v, z} d z d v \\
Q_{s}^{4}:=\int_{0}^{\theta_{s}} \int_{u}^{\theta_{s}} \int_{u}^{\theta_{v}}\left|\sigma_{z}^{\delta}-\sigma_{\theta_{z}}^{\delta}\right|(v-z)^{-\beta-1} d z d v(s-u)^{-\beta-1} d u \\
\leq C_{1}(\omega) \int_{0}^{\theta_{s}} \int_{u}^{\theta_{s}} \int_{u}^{\theta_{v}}(v-z)^{-\beta-1} d z d v(s-u)^{-\beta-1} d u \delta^{H-\rho} \\
\quad \leq C C_{1}(\omega) \delta^{H-\varepsilon-\beta}
\end{aligned}
$$

Substituting (38)-(41) into (37) yields

$$
\int_{0}^{\theta_{s}} \varphi_{s, u} d u \leq C_{1}(\omega)\left(C+C \delta^{\gamma-\beta}+C \int_{0}^{\theta_{s}}(s-v)^{-\beta} \int_{0}^{\theta_{v}} \varphi_{v, z} d z d v+C C_{1}(\omega) \delta^{H-\varepsilon-\beta}\right) .
$$

Then we apply an extension of the Gronwall inequality proved in [3]:

$$
\int_{0}^{\theta_{s}} \varphi_{s, u} d u \leq C_{I}(\omega) \exp C_{I I}(\omega) s
$$

where $C_{I}(\omega)=C_{2}(\omega)\left(C+C \delta^{\gamma-\beta}+C C_{1}(\omega)\right)$ and $C_{I I}(\omega)=C\left(C_{1}(\omega)+C\right)^{1 /(1-\beta)}$.

Now we show that the random variable $C_{I}(\omega) \exp C_{I I}(\omega) s$ possesses all the moments. Indeed,

$$
\mathrm{E} C_{I}(\omega)^{p} \exp p C_{I I}(\omega) s \leq\left(\mathrm{E} C_{I}(\omega)^{2 p}\right)^{1 / 2}\left(\mathrm{E} \exp 2 p C_{I I}(\omega) s\right)^{1 / 2}, \quad \mathrm{E} C_{I}(\omega)^{2 p}<\infty .
$$

Finally, we need to estimate $\operatorname{E} \exp \left(C \xi^{\varepsilon / 2(1-\beta)}\right)$, where

$$
\xi=\int_{0}^{T} \int_{0}^{T} \frac{\left|B_{x}^{H}-B_{y}^{H}\right|^{2 / \varepsilon}}{|x-y|^{2 H / \varepsilon}} d x d y
$$

Since $E \exp \lambda \xi^{\varepsilon \eta / 2}<\infty$ for all $\lambda>0$ and $0<\eta<2$ (see [11]) and since $1 /(1-\beta)<2$, all the moments of the random variable $C_{I}(\omega) \exp C_{I I}(\omega) s$ are finite.

Substituting bound (43) into (35), we prove the first inequality in (26). The second inequality in (26) follows from (30) and (36). The inspection of the above proof shows that the second moment is finite for the random variables in (29)-(43). Thus the second moment is finite for the random variables $C(\omega)$ involved in the inequalities (26).

Theorem 4.2 ([1, p. 249]). Let conditions 1)-7) hold for functions $a(t, x)$ and $b(t, x)$. Assume that the Hölder coefficient $\gamma$ in condition 6$)$ is such that $\gamma>H$ and that $\alpha=H-\frac{1}{2}$.

Then, for all $\rho \in(0,2 \alpha)$ and $\beta \in(1-H, 1 / 2)$, there exists a random variable $\widetilde{C}$ such that $\widetilde{C}(\omega)<\infty$ almost surely and

$$
U_{\delta}:=\sup _{0 \leq s \leq T}\left(\left|\sigma_{s}-\sigma_{s}^{\delta}\right|+\int_{0}^{\theta_{s}}\left|\triangle_{u, s}\left(\sigma, \sigma^{\delta}\right)\right|(s-u)^{-\beta-1} d u\right) \leq \widetilde{C}(\omega) \delta^{2 \alpha-\rho},
$$

where $\triangle_{u, s}\left(\sigma, \sigma^{\delta}\right):=\left|\sigma_{s}-\sigma_{s}^{\delta}-\sigma_{u}+\sigma_{u}^{\delta}\right|$; moreover $\widetilde{C}(\omega)$ does not depend on $\delta$.

Remark 4.1. As in the proof of Theorem 4.1, one can show that the second moment is finite for the random variable $\widetilde{C}(\omega)$.

Remark 4.2. Using Theorem 4.2, the preceding reasoning proves that $\left|\sigma_{s}-\sigma_{s}^{\delta}\right|<U_{\delta}$. Thus

$$
\mathrm{E}\left(\sigma_{s}-\sigma_{s}^{\delta}\right)^{2} \leq \mathrm{E} \widetilde{C}(\omega)^{2} \delta^{2(2 H-1)}=C \delta^{4 H-2} .
$$


This bound together with Theorems 3.1, 4.1, and 4.2 allows one to improve the rate of convergence for the approximating solution of equation (3) where the additional parameter is given by (22).

The rate of convergence for this case is

$$
\delta^{\min \{2 H-1, H, 1 / 2\}}=\delta^{\min \{2 H-1,1 / 2\}}
$$

if $H \in\left[\frac{1}{2}, 1\right]$. This means that the rate of convergence is $\delta^{2 H-1}$ if $H \in\left[\frac{1}{2}, \frac{3}{4}\right]$ and $\delta^{1 / 2}$ if $H \in\left[\frac{3}{4}, 1\right)$.

\section{BIBLIOGRAPHY}

1. Y. S. Mishura, Stochastic Calculus for Fractional Brownian Motion and Related Processes, Springer, 2008. MR 2378138(2008m:60064)

2. D. F. Kuznetsov, Stochastic Differential Equations: Theory and Practice of Numerical Solution, Sankt-Peterburgskii Gosudarstvennyi Politekhnicheskii Universitet, St. Petersburg, 2007. (Russian) MR2510680 (2010h:60175)

3. D. Nualart and A. Răşcanu, Differential equation driven by fractional Brownian motion, Collect. Math. 53 (2002), no. 1, 55-81. MR 1893308(2003f:60105)

4. P. Kloeden and E. Platen, Numerical Solution of Stochastic Differential Equations, Springer, 1999. MR 1214374 (94b:60069)

5. G. N. Milstein, Numerical Integration of Stochastic Differential Equations, Ural. Gos. Univ., Sverdlovsk, 1988; English transl., Kluwer Academic Publishers Group, Dordrecht, 1995. MR.1335454 (96e:65003)

6. V. S. Korolyuk and N. Limnios, Poisson approximation of increment processes with Markov switching, Theory Prob. Appl. (2005), no. 4, 629-644. MR2142564 (2006b:60183)

7. V. S. Korolyuk and N. Limnios, Compound Poisson Approximation with Drift for Stochastic Additive Functionals with Markov and Semi-Markov Switching, Springer, New York, 2005, pp. 279-297. MR2102958

8. V. S. Korolyuk and N. Limnios, Lévy approximation of increment processes with Markov switching, Stochastics Stochastics Rep. 76 (2004), no. 5, 383-394. MR.2096727 (2005h:60101)

9. V. V. Anisimov, Diffusion approximation in switching stochastic models and its application, Exploring Stochastic Laws, VSP, 1995, pp. 13-40. MR1713990(2001c:60142)

10. G. Kallianpur and R. Karandikar, Introduction to Option Pricing Theory, Birkhäuser, Boston, 2000. MR.1718056 (2000k:91054)

11. X. M. Fernique, Régularité des trajectoires de fonctions aléatoires gaussiennes, Lect. Notes in Math., vol. 480, Springer, Berlin, 1975. MR0413238 (54:1355)

Department of Probability Theory, Statistics, and Actuarial Mathematics, Faculty for Mechanics and Mathematics, National Taras Shevchenko University, Academician Glushkov Avenue 2, Kiev 03127, Ukraine

E-mail address: myus@univ.kiev.ua

Department of Probability Theory, Statistics, and Actuarial Mathematics, Faculty for Mechanics and Mathematics, National Taras Shevchenko University, Academician Glushkov Avenue 2, Kiev 03127, Ukraine

E-mail address: ShvajSS@ukr.net

Received 8/JUL/2009

Translated by S. KVASKO 